\title{
Bifurcation and chain recurrence
}

\author{
M. HURLEY \\ Department of Mathematics, Case Western Reserve University, Cleveland, \\ Ohio 44106, U.S.A. \\ (Received 5 February 1982)
}

Abstract. We show that there is a residual subset of the set of $C^{1}$ diffeomorphisms on any compact manifold at which the map

$$
f \rightarrow(\text { number of chain components for } f)
$$

is continuous. As this number is apt to be infinite, we prove a localized version, which allows one to conclude that if $f$ is in this residual set and $X$ is an isolated chain component for $f$, then

(i) there is a neighbourhood $U$ of $X$ which isolates it from the rest of the chain recurrent set of $f$, and

(ii) all $g$ sufficiently $C^{1}$ close to $f$ have precisely one chain component in $U$, and these chain components approach $X$ as $g$ approaches $f$.

(ii) is interpreted as a generic non-bifurcation result for this type of invariant set.

\section{0 . Introduction}

A classical set of problems in the study of dynamical systems is concerned with understanding the structure of various invariant sets of a given system, and to describe how these sets change as one changes the system. This bifurcation problem is well understood in some instances. For example, the theorems of Kupka \& Smale and Hartman \& Grobman tell us that for each $f$ in a residual subset of $\operatorname{Diff}^{r}(M)(r \geq 1)$, and each $n \geq 1, f^{n}$ has a finite number of fixed points, and that if $g$ is $C^{r}$ close enough to $f$, (how close depends on $n$ ), then $g^{n}$ has exactly the same number of fixed points as $f^{n}$, and the fixed point set of $g^{n}$ approaches that of $f^{n}$ as $g$ approaches $f$. See $[\mathbf{6}]$ or $[\mathbf{8}]$ for more details.

The main result of the present paper is an analogue of this well-known result. The setting is a compact Riemannian manifold $M$, with metric $d$. We consider the chain recurrent set of a map $f$ in $\operatorname{Diff}^{r}(M), r \geq 0$. (Diff $(M)$ is the set of homeomorphisms of $M$ to itself with the uniform metric $d_{0}$; Diff ${ }^{1}(M)$ is the set of $C^{1}$ diffeomorphisms on $M$ with the uniform $C^{1}$ metric $d_{1}$, and so on.)

A point $x$ in $M$ is $\alpha$-chain recurrent for $f$ if for each $\beta>\alpha \geq 0$ there is a $\beta$-chain from $x$ back to $x$, that is, a finite set of points

$$
x_{0}, x_{1}, \ldots, x_{p}, \quad \text { with } x=x_{0}=x_{p},
$$

and

$$
d\left(f\left(x_{i}\right), x_{i+1}\right)<\beta \quad \text { for } i=0,1,2, \ldots, p-1 .
$$


We will sometimes represent such a chain by the notation

$$
x_{0} \rightarrow x_{1} \rightarrow \cdots \rightarrow x_{p}
$$

and say that this $\beta$-chain goes from $x_{0}$ to $x_{p}$.

The chain recurrent set of $f, \mathrm{CR}(f)$, is the set of points in $M$ that are $\alpha$-chain recurrent for all $\alpha \geq 0$, (so chain recurrent and 0 -chain recurrent mean the same thing). There is a natural equivalence relation that is defined on $\operatorname{CR}(f)$ by calling two points equivalent if for any $\beta$ there is a periodic $\beta$-chain containing both points. More precisely, $x \sim y$ if for each $\beta>0$ there is a $\beta$-chain going from $x$ to $y$ and a $\beta$-chain going from $y$ to $x$. Each equivalence class is called a chain component. Let $N(f)$ denote the (possibly infinite) number of chain components of $f$.

The basic new result of this paper is part (b) of the following theorem. Recall that a subset $S$ of a topological space $X$ is residual if $S$ can be realized as a countable intersection of open, dense subsets of $X$.

THEOREM A. There is a residual subset I of $\operatorname{Diff}^{1}(M)$ such that whenever $f$ is an element of $I$, then

(a) (Conley, Takens) $f$ is a continuity point of $\mathrm{CR}\left(\_\right)$;

(b) $N($ ) is continuous at $f$.

One interprets the continuity in part (a) of the theorem by viewing $\mathrm{CR}\left(\_\right)$as a map from $\left(\operatorname{Diff}^{1}(M), d_{1}\right)$ to $\left(\mathrm{FM}, d_{H}\right)$, where $d_{1}$ is the uniform $C^{1}$ metric on Diff $^{1}(M), F M$ is the set of all closed non-void subsets of $M$, and $d_{H}$ is the Hausdorff metric on FM,

$$
d_{H}\left(F, F^{\prime}\right)=\inf \left\{t>0 \mid F^{\prime} \subset B(F, t) \text { and } F \subset B\left(F^{\prime}, t\right)\right\},
$$

where

$$
B(F, t)=\{x \in M \mid d(x, F)<t\} .
$$

The relevant facts are that $\left(\operatorname{Diff}^{1}(M), d_{1}\right)$ is a Baire space [2], so that any residual subset is dense; and that the metric topology makes (FM, $\left.d_{H}\right)$ a compact metric space [4]. A more detailed description is contained in [3]. The proof of (a) is essentially due to C. Conley. His description of CR $(f)$ in terms of the attractors of $f$ (see II.6 and II.7 of [1], especially 6.2.A on page 37) shows that the map $f \rightarrow \mathrm{CR}(f)$ is upper semicontinuous; from this it is a standard argument to establish (a). More details are contained in the discussion surrounding lemma 1, below. See also [10, theorem 1], and corollary $3(\mathrm{a})$, below.

In part (b) of the theorem, the range of the map $N()$ is $\{1,2, \ldots, \infty\}$ viewed as the usual one-point compactification of the positive integers.

Theorem A more or less provides the kind of non-bifurcation result we spoke of in the opening paragraph of this paper. If $\left.N()_{-}\right)$is continuous and finite at $f$, then diffeomorphisms that are $C^{1}$ close to $f$ have exactly the same number of chain components as does $f$. In other words, one cannot break apart any of the chain components of $f$ by using a $C^{1}$ perturbation. Unfortunately, the conclusion that one can draw from the continuity of $N($ ) at $f$ is much less informative if $N(f)$ is infinite. Of course, this would not be a problem if $N(g)$ were finite for all $g$ in 
a residual subset of $\operatorname{Diff}^{1}(M)$, but it is not known whether or not this is the case (unless the dimension of $M$ is small). Moreover, S. Newhouse has shown, [5], that any residual subset of $\operatorname{Diff}^{r}(M)$ must contain diffeomorphisms $f$ with $N(f)=\infty$ whenever both $r$ and the dimension of $M$ are at least 2. This fact motivates a different approach to improving the conclusions that can be drawn when $N(f)$ is infinite. Instead of viewing the dynamics on all of $M$, we can consider only the chain-recurrent behaviour that is contained in a specified subset $Y$ of $M$. Specifically, we say that an $\alpha$-chain

$$
x_{0} \rightarrow x_{1} \rightarrow \cdots \rightarrow x_{p}
$$

is an $(\alpha, Y)$-chain if each $x_{i}$ lies in the closure of $Y$. CR $(f ; Y)$ is then defined to be the set of points $x$ such that for each $\alpha>0$ there is an $(\alpha, Y)$-chain from $x$ back to $x$,

$$
x=x_{0} \rightarrow x_{1} \rightarrow \cdots \rightarrow x_{p}=x .
$$

Just as in the original case, one can say that $x$ and $y$ in $\mathrm{CR}(f ; Y)$ are equivalent if for each $\alpha>0$ there is a periodic $(\alpha, Y)$-chain

$$
x_{0} \rightarrow \cdots \rightarrow x_{p}=x_{0} \quad \text { with } x=x_{0} \quad \text { and } \quad y=x_{i} \quad \text { for some } i .
$$

An equivalence class under this relation will be called a $Y$-chain component of $f$. Let $N(f ; Y)$ denote the (again, possibly infinite) number of $Y$-chain components of $f$.

THEOREM B. There is a countable basis $U$ for the topology on $M$, and a residual subset $J$ of $\operatorname{Diff}^{1}(M)$ with the property that whenever $f$ is an element of $J$ and $U$ is an element of $U$, then

(a) $f$ is a continuity point of $\mathrm{CR}(; U)$;

(b) $N($; U ) is continuous at $f$.

Note that by taking $U=M$ in theorem B one obtains theorem $\mathrm{A}$.

Corollary. Suppose $f$ is in $J, X$ is a chain component of $f$, and $B$ is an open neighbourhood of $\boldsymbol{X}$ with

$$
\operatorname{clos}(B) \cap \operatorname{CR}(f)=X
$$

Then there is a neighbourhood $G$ of $f$ in $\operatorname{Diff}^{1}(M)$ such that each $g$ in $G$ has exactly one chain component $X_{\mathrm{g}}$ contained in $B$, and no other chain component of $\mathrm{g}$ meets $\operatorname{clos}(B)$.

The proof of theorem B relies on the affirmative answer to the following stabilization question for invariant sets:

If $U$ is open in $M$ and contains an $f$-invariant set, can $f$ be $C^{r}$ approximated by $g$ such that any diffeomorphism sufficiently $C^{r}$ close to $g$ has an invariant set contained in $U$ ?

We use the closing lemma [7] to obtain an affirmative answer to this question. This is the reason we restrict ourselves to $C^{1}$ diffeomorphisms in theorems $\mathbf{A}$ and $\mathbf{B}$. 
As an application of the above results, we obtain the following theorem. To say that $A$ in $M$ is a chain transitive attractor of $f$ means that

(1) $A$ is a chain component of $f$;

(2) $\boldsymbol{A}$ is an attractor of $f$; that is, there is an open neighbourhood $U$ of $A$ with $f($ clos $(U))$ contained in $U$ and

$$
\bigcap_{n \geq 0} f^{n}(U)=A
$$

THEOREM C. There is a residual subset $\mathscr{A}$ of $\operatorname{Diff}^{1}(M)$ such that if $f$ is in $\mathscr{A}, f_{n}$ converges to $f$ in the $C^{1}$ topology, and $A$ is a chain transitive attractor of $f$, then there are chain transitive attractors $A_{n}$ of $f_{n}$ with $A_{n}$ converging to $A$ in the Hausdorff topology. For large $n$ there is only one $A_{n}$ that is near $A$.

The $C^{r}$ version of theorem $C$ was stated in [3] (theorem $A(c)$ ). However, the proof there contains an error. The $C^{r}$ version of theorem $C$ for $r$ greater than 1 is still an unsettled question.

$\S 1$ contains the proof of theorem $B$ as well as a study of the stabilization question that is the key to that result. $\S 2$ contains the application to attractors.

\section{Proof of theorem $B$}

We begin with three simple lemmas.

LemMA 1. Suppose $X_{1}$ and $X_{2}$ are metric spaces, with $X_{2}$ compact. Let $h: X_{1} \rightarrow F X_{2}$ be either upper or lower semicontinuous. Then the set of continuity points of $h$ is $a$ residual subset of $X_{1}$.

This is [9, lemma 2.3]. The map $h$ is lower semicontinuous at $x$ in $X_{1}$ if whenever $x_{n}$ approaches $x$ and $y$ is in $h(x)$, then there are points $y_{n}$ in $h\left(x_{n}\right)$ with $y_{n}$ converging to $y$. $h$ is upper semicontinuous at $x$ if for any sequence $x_{n} \rightarrow x$, if $y_{n}$ is in $h\left(x_{n}\right)$ and $y_{n} \rightarrow y$, then $y$ is in $h(x)$. If $g$ is a map from some topological space $X_{1}$ into the extended half-line $S=[0, \infty]$ (viewed as the one-point compactification of $[0, \infty)$ ), then $g$ is lower (upper) semicontinuous at $x$ in $X_{1}$ if and only if the induced map

$$
g^{*}: X_{1} \rightarrow \text { FS given by } g^{*}(z)=[0, g(z)]
$$

is lower (upper) semicontinuous at $x$. This agrees with the usual definition of lower (upper) semicontinuity for real-valued functions:

$$
\liminf _{z \rightarrow x} g(z) \geq g(x) \quad\left(\limsup _{z \rightarrow x} g(z) \leq g(x)\right) .
$$

LeMMA 2. Fix $f$ in $\operatorname{Diff}^{r}(M)$ and a closed subset $Y$ of $M$. Define a map $G: Y \rightarrow[0, \infty)$ by

$$
\begin{gathered}
G(x)=\inf \{\alpha>0 \mid x \text { lies on a periodic } \alpha \text {-chain for } f \text { which } \\
\text { is contained in } Y\} .
\end{gathered}
$$

Then $G$ is continuous.

Proof. Let $\gamma>0$ be given, and choose $\delta<\gamma$ small enough that whenever $x, y$ are in $M$ and $d(x, y)<\delta$ then

$$
d(f(x), f(y))<\gamma
$$


Now suppose that $x, y$ are in $Y$ and within $\delta$ of each other, and that

$$
x \rightarrow x_{1} \rightarrow \cdots \rightarrow x_{p} \rightarrow x
$$

is an $\alpha$-chain for $f$ in $Y$. Then

and

$$
d\left(f(y), x_{1}\right) \leq d(f(y), f(x))+d\left(f(x), x_{1}\right)<\gamma+\alpha,
$$

$$
d\left(f\left(x_{p}\right), y\right) \leq d\left(f\left(x_{p}\right), x\right)+d(x, y)<\alpha+\gamma .
$$

Thus $d(x, y)<\delta$ implies that

$$
G(y) \leq G(x)+\gamma .
$$

By the symmetry in the assumptions on $x$ and $y$ we conclude that

$$
|G(x)-G(y)|<\gamma
$$

whenever $d(x, y)<\delta$, so $G$ is continuous.

Note that for $Y$ closed in $M$ and $G$ defined as in the lemma,

$$
\mathrm{CR}(f ; Y)=G^{-1}(0),
$$

so the lemma shows that CR $(f ; Y)$ is closed in $M$. In addition, it shows that distinct $Y$-chain components of $f$ are bounded apart in the sense that if $X_{1}$ and $X_{2}$ are disjoint $Y$-chain components of $f$ then for some $\alpha>0$ there is no periodic $\alpha$-chain for $f$ that is both contained in $Y$ and meets each of the sets $X_{1}$ and $X_{2}$.

LEMMA 3. Suppose $f_{n}$ converges to $f$ in the $C^{0}$ topology, $y_{n} \rightarrow y$ and $z_{n} \rightarrow z$ in $M$, and that for each $n$ and each positive $\alpha$ there is an $(\alpha, Y)$-chain for $f_{n}$ going from $y_{n}$ to $z_{n}$. Then for each positive $\alpha$ there is an $(\alpha, Y)$-chain for $f$ going from $y$ to $z$.

Proof. Since $(\alpha, Y)$-chains and $(\alpha, \operatorname{clos}(Y))$ - chains are the same thing, we may as well assume that $Y$ is closed. Let $\alpha>0$ be given. Choose $n$ large enough that

(i) $d_{0}\left(f, f_{n}\right)<\alpha / 3$;

(ii) $d\left(f(y), f\left(y_{n}\right)\right)<\alpha / 3$;

(iii) $d\left(y, y_{n}\right)<\alpha / 3$;

(iv) $d\left(z, z_{n}\right)<\alpha / 3$.

By assumption there is an $(\alpha / 3, Y)$-chain for $f_{n}$ that goes from $y_{n}$ to $z_{n}$; denote it by

$$
y_{n}=x_{0} \rightarrow x_{1} \rightarrow \cdots \rightarrow x_{p}=z_{n}
$$

(of course the $x_{j}$ 's depend on $n$, but we suppress this dependence from the notation). Now (i)-(iv) combined with the triangle inequality show that

$$
y \rightarrow x_{1} \rightarrow x_{2} \rightarrow \cdots \rightarrow x_{p-1} \rightarrow z
$$

is a $\alpha$-chain in $Y$ for $f$.

Corollary 3(a). The map $f \rightarrow \mathrm{CR}(f ; Y)$ is upper semicontinuous at all $f$ in $\operatorname{Diff}^{r}(M)$, for any $r \geq 0$ and any subset $Y$ of $M$.

Corollary 3(b). If $f, f_{n}, y, y_{n}, z, z_{n}$ are as in lemma 3 and for each $n, y_{n}$ and $z_{n}$ lie in a single $Y$-chain component of $f_{n}$, then $y$ and $z$ lie in a single $Y$-chain component of $f$. 
Proof. 3(a), in the case $Y=M$, is essentially (although not explicitly) contained in [1]; see also [10] for a very closely related result. To prove 3(a) just use the lemma with $y_{n}=z_{n}$ for each $n$.

As for 3(b), the assumptions ensure that for each $n$ and each positive $\alpha$ there are $(\alpha ; Y)$-chains for $f_{n}$ from $y_{n}$ to $z_{n}$ and from $z_{n}$ to $y_{n}$. Applying the lemma twice gives $(\alpha ; Y)$-chains for $f$ from $y$ to $z$ and from $z$ to $y$, which establishes 3(b).

Proposition 4. The set

$$
\mathscr{C}_{r}(Y)=\left\{f \in \operatorname{Diff}^{r}(M) \mid \mathrm{CR}\left(\_; Y\right) \text { is continuous at } f\right\}
$$

is residual in $\operatorname{Diff}^{r}(M)$ for any subset $Y$ of $M$, and $r$ in $\{0,1, \ldots, \infty\}$.

Proof. Combine lemma 1 and corollary 3(a).

Recall that $N(f ; Y)$ is the number of $Y$-chain components of $f$.

Proposition 5. Suppose $f$ is in $\mathscr{C}_{r}(Y)$. Then $N(, ; Y)$ is lower semicontinuous at $f$.

Proof. Let $f_{n}$ approach $f$ in $\operatorname{Diff}^{r}(M)$. Suppose first that $N(f ; Y)$ in finite, so that we can list the $Y$-chain components, $X_{1}, \ldots, X_{k}$. Since these sets are closed and disjoint, we can find open sets $G_{1}, \ldots, G_{k}$ (open in $Y$ ) such that $X_{j}$ is contained in $G_{j}$ and the closures of the sets $G_{j}$ are pairwise disjoint. Since $\operatorname{CR}\left(f_{n} ; Y\right)$ is assumed to approach $\mathrm{CR}(f ; Y)$ in the Hausdorff topology, we can conclude that, for large $n$, at least one $Y$-chain component of $f_{n}$ meets each of the sets $G_{j}$. We can then apply corollary 3 (b) to see that for large enough $n$ no $Y$-chain component of $f_{n}$ can meet more than one of the sets $G_{j}$. Thus

$$
\lim \inf N\left(f_{n} ; Y\right) \geq \text { number of } G_{j} \text { 's }=k=N(f ; Y) .
$$

Now suppose that $N(f ; Y)$ is infinite. By arguing as above for arbitrary finite collections of $Y$-chain components of $f$, we can show that $\lim \inf N\left(f_{n} ; Y\right)$ is also infinite.

In what follows it will become necessary to restrict the possible subsets $Y$ that we will consider. Accordingly, we fix a certain countable basis $\mathcal{U}$ for the topology on $M$. We require that $\mathscr{U}$ contains enough open sets so that any two disjoint, closed subsets of $M$ can be separated by elements $U, U^{\prime}$ of $\mathcal{U}$ with

$$
\operatorname{clos}(U) \cap \operatorname{clos}\left(U^{\prime}\right)=\varnothing .
$$

We àlso require that each element of $\mathcal{U}$ be an open subset of $M$ whose topological boundary is a smooth codimension-one submanifold of $M$. Obtaining such a basis is no problem, since a compact manifold is always second countable, and obtaining the smoothness condition involves only some elementary arguments in differential topology (see, e.g. [2, exercise 1, p. 55]). This smoothness condition will facilitate certain technical arguments, and it does not interfere with the applications we have in mind (specifically, the corollary to theorem $\mathrm{B}$, and theorem $\mathrm{C}$ ).

Define $\mathscr{C}_{r}$ to be the intersection of all the sets $\mathscr{C}_{r}(U)$ for $U$ in $\mathscr{U}$. By proposition 4 and the fact that $\mathscr{U}$ is countable, $\mathscr{C}_{r}$ is a residual subset of $\operatorname{Diff}^{r}(M)$. 
THEOREM 6. There is a residual subset $J$ of $\operatorname{Diff}^{1}(M)$ with the property that whenever $f$ is in $J$ and $U$ is in $U$, then

(a) $f$ is a continuity point of $\mathrm{CR}\left(\_; U\right)$;

(b) $N\left(\_, U\right)$ is continuous at $f$.

Proposition 5 and lemma 1 combine to give a residual subset $J$ of $\mathscr{C}_{1}$ at which the restriction of $N(; U)$ to $\mathscr{C}_{1}$ is continuous for each $U$ in $\mathscr{U}$. Since a residual subset of a residual set is residual, $J$ is residual in $\operatorname{Diff}^{1}(M)$. We need to show that the unrestricted map $N\left(\_; U\right)$ is continuous at any $f$ in $J$. If $N(f ; U)$ is infinite, this is equivalent to the lower semicontinuity of proposition 5 , so we may assume that $f_{n}$ approaches $f$ in $\operatorname{Diff}^{1}(M)$ with

$$
\infty \geq N\left(f_{n} ; U\right)>N(f ; U) \quad \text { for all } n,
$$

and look for a contradiction. By assumption on $f$, there is a $\delta>0$ such that $d_{1}(f, g)<\delta$ and $g$ in $\mathscr{C}_{1}$ imply that $N(g ; U)$ is equal to $N(f ; U)$, so to get a contradiction it will suffice to show that we can perturb any $f_{n}$ to a map $g_{n}$ in $\mathscr{C}_{1}$ with

$$
N\left(g_{n} ; U\right) \geq N\left(f_{n} ; U\right) .
$$

This is a type of stabilization problem; it may be phrased in a stronger way as follows.

Stabilization question. If $X$ is a $U$-chain component for $f$, is there an open set $G$ in $\operatorname{Diff}^{r}(\boldsymbol{M})$, containing $f$ in its closure, such that every $g$ in $G$ has at least one $U$-chain component?

By using the closing lemma [7], we can give an affirmative answer in the case $r=1$.

Closing Lemma (Pugh). Suppose that $\left\{f^{n}(x) \mid-\infty<n<\infty\right\}$ is a recurrent orbit for $a C^{1}$ diffeomorphism $f$, that $V$ is an open set containing the closure of this orbit, and that $\delta>0$. Then there is a diffeomorphism $g, C^{1}$ - $\delta$-close to $f$, with a hyperbolic periodic orbit in $V$.

Proof. The proof is contained in [7], although the statement that the periodic orbit lies in $V$ is not explicitly made there. The argument in [7] proceeds by choosing a finite segment of the recurrent orbit, and then making perturbations in a small neighbourhood, $W$, of this orbit segment. The closed orbit that is produced consists of two segments, the first contained in $W$, and the second being a segment of the original recurrent orbit. Hence one only has to ensure that $W$ is contained in $V$. For further details and for the definitions of 'hyperbolic' and 'recurrent', see [7] or [8].

LEMMA 7. Let $U$ be an element of $U$, $f$ be in $\operatorname{Diff}^{1}(M)$, and suppose $N(f ; U) \geq 1$. Then there is an open set $W$ in $\operatorname{Diff}^{1}(M)$ with $f$ in the closure of $W$ and $N(g ; U) \geq 1$ for all $\mathrm{g}$ in $W$.

Proof. $N(f ; U) \geq 1$ implies that CR $(f ; U)$ is a non-void invariant set in the closure of $U$, so that clos $(U)$ contains a recurrent orbit $\left\{f^{n}(x)\right\}$. The smoothness conditions on $U$ in $U$ ensure that there is a smooth diffeomorphism $h, C^{1}$-close to the identity, with $h(\operatorname{clos}(U))$ contained in $U$. If we let $y=h(x)$ then $y$ is a recurrent point of 
the diffeomorphism

$$
g_{0}=h \circ f \circ h^{-1},
$$

and the closure of the $g_{0}$-orbit of $y$ lies in $U$. By the closing lemma, we can find $g C^{1}$-close to $g_{0}$ such that $g$ has a hyperbolic periodic orbit contained in $U$. By the local stability of hyperbolic orbits (see [6] for example), there is an open neighbourhood $W(\mathrm{~g})$ of $g$ such that each map in $W(\mathrm{~g})$ also has a periodic orbit in $U$. Let $W$ be the union of $W\left(g_{n}\right)$ for a sequence $g_{n}$ converging to $f$.

Because the perturbations involved in the proof of lemma 7 are local (that is, they can be required to be the identity away from $U$, we obtain as an immediate corollary:

Proposition 8. Let $\delta>0$ be given, and suppose that $X_{1}, \ldots, X_{k}$ are distinct $U$-chain components of $h(U$ in $\mathcal{U})$, with each $X_{j}$ contained in an open set $U_{j}$ in $U, U_{j} \subset U$, with the closures of the various $U_{j}$ 's pairwise disjoint. Then there is a $g$ in $\mathscr{C}_{1}$ with $d_{1}(h, g)<\delta, \operatorname{CR}\left(g ; U_{j}\right)$ non-void for each $j$, and $N(g ; U) \geq k$.

Proof of theorem 6. Part (a) of the theorem follows from proposition 4 and the fact that $\mathscr{U}$ is countable, so we turn our attention to the proof of (b). $f$ in $J$ implies that for all $g$ in a neighbourhood of $f$ in $\mathscr{C}_{1}$

$$
N(g ; U)=N(f ; U)
$$

(recall that we can assume that $N(f ; U)$ in finite). Since $J$ is contained in $\mathscr{C}_{1}$, for all $h$ in a $C^{1}$ neighbourhood of $f$

$$
N(h ; U) \geq N(f ; U) .
$$

If one could find $h$ arbitrarily close to $f$ with strict inequality in $\left(^{* *}\right)$, proposition 8 would allow one to find $g$ in $\mathscr{C}_{1}$, arbitrarily close to $f$, with

$$
N(g ; U) \geq N(h ; U)>N(f ; U) .
$$

This would contradict $\left({ }^{*}\right)$, and so the theorem is established.

Using theorem 6 and the upper semicontinuity of $\mathrm{CR}()$, it is not hard to see that if $X$ is an isolated chain component of $f$ in $J$ and $g$ is $C^{1}$ close to $f$, then the single local chain component counted by $N(g ; U)$ (here $U$ in $\mathscr{U}$ is an open set that separates $X$ from the rest of $\mathrm{CR}(f))$ is in fact a full chain component of $g$. In other words, if $U$ is in $\mathscr{U}$ and

$$
\mathrm{CR}(f) \cap U=\mathrm{CR}(f) \cap \operatorname{clos}(U)
$$

is a single chain component of $f$, then

$$
\mathrm{CR}(g ; U)=\mathrm{CR}(g) \cap U
$$

is a single chain component of $g$ for all $g C^{1}$ close to $f$.

\section{Proof of theorem $C$}

Recall that the definition of 'chain transitive attractor' was given in the introduction.

THEOREM 9. There is a residual subset $\mathscr{A}$ of $\operatorname{Diff}^{1}(M)$ such that for any $f$ in $\mathscr{A}$ and any sequence $f_{n}$ which converges to $f$ in the $C^{1}$ topology, and any chain transitive attractor $A$ of $f$, there are attractors $A_{n}$ of $f_{n}$ with $A_{n}$ converging to $A$ in the Hausdorff 
topology. Moreover, for all large $n$ there is a unique choice of $\boldsymbol{A}_{n}$, and it will be chain transitive for $f_{n}$.

The uniqueness in the statement of the theorem is to be interpreted as follows. There are neighbourhoods $V$ of $A$ and $W$ of $f$ such that whenever $f_{n}$ is in $W$, then $A_{n}$ can be chosen so that $A_{n}$ is in $V$. There is only one such choice, and it is a chain transitive attractor. Since $A_{n}$ converges to $A$, this choice must be made for all large $n$.

LeMMA 10. Suppose $g$ is in $\operatorname{Diff}^{1}(M)$ and that $A_{1}$ and $A_{2}$ are attractors of $g$ with $A_{1} \neq A_{2}$. Then $A_{1} \cup A_{2}$ contains at least two chain components of $g$.

Proof. Recall that the $\alpha$-limit set of $x$ under $g$

$$
\alpha(x)=\bigcap_{m<0} \operatorname{clos}\left\{g^{i}(x) \mid i \leq m\right\},
$$

is closed, non-empty, and $g$-invariant. It is easy to see that $\alpha(x)$ is also chain transitive ([1, II.4 and II.6.2]). It follows that any compact, non-void, $g$-invariant set contains at least one chain component. Consequently we need only show that $A_{1} \neq A_{2}$ forces the existence of two disjoint, compact, non-empty, $g$-invariant sets in the union of $A_{1}$ and $A_{2}$. If $A_{1}$ and $A_{2}$ are disjoint this is immediate, so assume that

$$
A_{3}=A_{1} \cap A_{2} \neq \varnothing
$$

By [1, II.5.3.A $]_{2} A_{3}$ is also an attractor of $g$. Let $x$ be in $A_{1}-A_{3}$ and consider $\alpha(x)$. Since $A_{1}$ is compact and $g$-invariant, $\alpha(x)$ is closed, non-empty, $g$-invariant, and in $A_{1}$. Since $A_{3}$ is an attractor, it is not hard to show that $\alpha(x) \cap A_{3}$ is non-empty if and only if $x$ is in $A_{3}$ (see [1, II.5.1.A]). Since we are assuming that $x$ is not in $A_{3}$, we must conclude that $\alpha(x)$ is contained in $A_{1}-A_{3}$. Hence $\alpha(x)$ and $A_{3}$ are the disjoint, non-empty, compact, $g$-invariant sets we require.

Proof of theorem 9. By [3, 7.15], there is a residual subset $T$ of $\operatorname{Diff}^{1}(M)$ satisfying all but the uniqueness part of theorem 9. Let $\mathscr{A}$ be the intersection of $T$ with the residual set $J$ of theorem 6 . Since $A$ is a chain transitive attractor of $f$, we can find $U$ in $U$ with $N(f ; U)=1$, so $N(g ; U)=1$ for all $g C^{1}$ close to $f$. Thus, if $g$ is near $f$, then $g$ has exactly one attractor in $U$, for if there were more than one, then the lemma shows that $g$ would also have more than one chain component in $U$. Let $A_{g}$ denote this uniquely defined attractor of $g$. Since $A_{g}$ is an attractor none of whose proper subsets is an attractor, $A_{g}$ is chain transitive (this follows from Conley's characterization of CR $(g)$ in terms of the attractors of $g$; see $[1,6.2 . A, p .37])$.

\section{REFERENCES}

[1] C. Conley. Isolated Invariant Sets and the Morse Index. Amer. Math. Soc.: Providence, 1978.

[2] M. Hirsch. Differential Topology. Springer-Verlag: New York, 1976.

[3] M. Hurley. Attractors: persistence, and density of their basins. Trans. Amer. Math. Soc. 269 (1982), 247-271.

[4] S. Nadler. Hyperspaces of Sets. Marcel Dekker: New York, 1978. 
[5] S. Newhouse. Diffeomorphisms with infinitely many sinks. Topology. 13 (1974), 9-18.

[6] Z. Nitecki. Differentiable Dynamics. M.I.T. Press; Cambridge, 1971.

[7] C. Pugh. The closing lemma. Amer. J. of Math. 89 (1967), 956-1009.

[8] S. Smale. Differentiable dynamical systems. Bull. Amer. Math. Soc. 73 (1967), 747-817.

[9] F. Takens. On Zeeman's tolerance stability conjecture. Manifolds-Amsterdam, 1970, Springer Lecture Notes in Math. No. 197. Springer-Verlag: New York, 1971.

[10] F. Takens. Tolerance stability. Dynamical Systems-Warwick, 1974, Springer Lecture Notes in Math. No. 468, pp. 293-304. Springer-Verlag: New York, 1975. 